\title{
A Biomimetic Enantioselective Total Synthesis of (-)-Siccanin by The Pd-Catalyzed Asymmetric Allylic Alkylation (AAA) And Sequential Radical Cyclizations
}

\author{
Barry M. Trost, Hong C. Shen and Jean-Philippe Surivet \\ Department of Chemistry \\ Stanford University \\ Stanford, CA 94305-5080
}

\section{Supplemental Information}

\section{Experimental}

\section{General}

All reactions were performed under a nitrogen atmosphere unless otherwise indicated. Some solvents were freshly purified by column before use: acetonitrile, methylene chloride, toluene and diethyl ether. Other solvents were purified by distillation: benzene, triethylamine, and pyridine from calcium hydride; tetrahydrofuran, dioxane from sodium benzophenone ketyl; and acetone from calcium sulfate; and dimethylformamide from barium oxide and stored over $4 \AA$ molecular sieves. Methanol was distilled from magnesium methoxide. Tris(dibenzylideneacetone)palladium(0) monochloroform complex, $\mathrm{Pd}_{2} \mathrm{dba}_{3} \cdot \mathrm{CHCl}_{3}$ was prepared by the procedure of Ibers ${ }^{1}$. All other reagents were used as obtained unless otherwise noted.

Flash Chromatography was performed with EM Science silica gel (0.040-0.063 $\mu \mathrm{m}$ grade). Analytical thin-layer chromatography was performed with $0.2 \mathrm{~mm}$ coated commercial silica gel plates (E. Merck, DC-Plasrikfolien, kieselgel $60 \mathrm{~F}_{254}$ ). Melting

\footnotetext{
${ }^{1}$ Ukai, T.; Kawazura, H.; Iishii, Y.; Bonnett, J.J.; Ibers, J.A. J. Organomet. Chem. 1974, 65, 263.
} 
points were obtained on a Thomas-Hoover apparatus in open capillary tubes and are uncorrected. Kugelrohr distillations were performed on a Büchi GKR-50 glass tube oven. Proton nuclear magnetic resonance ( ${ }^{1} \mathrm{H}-\mathrm{NMR}$ ) data were acquired at $300 \mathrm{MHz}$ on a Varian GEM-300 and at $500 \mathrm{MHz}$ on a Varian GEM-500 spectrometer. Chemical shifts are reported in delta $(\delta)$ units, in parts per million (ppm) downfield from tetramethylsilane, of in ppm relative to the singlet at $7.26 \mathrm{ppm}$ from chloroform- $d$. Splitting patterns are designated as s, singlet; $d$, doublet; $t$, triplet; $q$, quartet; $p$, pentet, $m$, multiplet, br, broad. Carbon-13 nuclear magnetic resonance $\left({ }^{13} \mathrm{C}-\mathrm{NMR}\right)$ data were acquired at $75 \mathrm{MHz}$ on a Varian GEM-300 and at $125 \mathrm{MHz}$ on a Varian GEM-500 spectrometer. Chemical shifts are reported in ppm relative to the center line of a triplet at $77.0 \mathrm{ppm}$ for choroform- $d$ and the center line of a septet at $39.5 \mathrm{ppm}$ in dimethylsulfoxide- $d_{6}$.

Infrared (IR) data were recorded as films on sodium chloride plates or a potassium bromide (KBr) pellets on a Nicolet 205 and Perkin-Elmer Paragon 500 FT-IR spectrometers. Absorbance frequencies are reported in reciprocal centimeters $\left(\mathrm{cm}^{-1}\right)$. High resolution mass spectral data (HRMS) were obtained from Mass Spectrometry Resource, School of Pharmacy, University of California-San Francisco, on a Kratos MS9 spectrometer at an ionizing current of $98 \mathrm{~mA}$ and an ionizing voltage of $70 \mathrm{eV}$. Elemental analyses (Anal.) were performed by M.-H.-W. Laboratories of Pheonix, AZ. Chiral HPLC analyses were performed on a Spectra Series P-100 chromatograph using the chiral column and heptane:iso-propanol mixtures indicated. Optical rotations were measured on a Jasco DIP-1000 digital polarimeter using $5 \mathrm{~cm}$ cells and the sodium D line $(589 \mathrm{~nm})$ at ambient temperature in the solvent and concentration indicated.

(2,2-Dimethyl-6-methylenecyclohexyl)-acetic acid 2-thioxo-2H-1-pyridinyl ester (29): Method A: ${ }^{2}$ To a solution of carboxylic acid $27(160 \mathrm{mg}, 0.88 \mathrm{mmol})$ in $5 \mathrm{~mL}$ of distilled THF were added $N$-methylmorpholine $(98 \mathrm{mg}, 0.97 \mathrm{mmol})$ and isobutylchloroformate (132 $\mathrm{mg}, 0.97 \mathrm{mmol})$ at $-20^{\circ} \mathrm{C}$. After $20 \mathrm{~min}$ at this temperature, to the reaction mixture was added $N$-hydroxythiopyridone sodium salt $(158 \mathrm{mg}, 1.06 \mathrm{mmol})$. This reaction was kept at $-10^{\circ} \mathrm{C}$ for $10 \mathrm{~h}$. Withtout workup, the solution was purified by flash

\footnotetext{
${ }^{2}$ Schneider, C.; Kazmaier, U. Eur. J. Org. Chem. 1998, 1155.
} 
chromatography eluting with $5 \%$ to $30 \%$ diethyl ether in petroleum ether to afford hydroxyester 29 (133 mg, $0.46 \mathrm{mmol}, 52 \%$ ) as a yellow oily solid.

Method B: ${ }^{3}$ To a solution of 2-mercaptopyridine $N$-oxide (26 mg, $0.20 \mathrm{mmol}$ ) and 1-(3dimethylaminopropyl)-3-ethylcarbondiimide hydrochloride $(47 \mathrm{mg}, 0.24 \mathrm{mmol})$ in 0.5 $\mathrm{mL}$ of dichloromethane at $0^{\circ} \mathrm{C}$ was added a solution of carboxylic acid $27(37 \mathrm{mg}, 0.203$ mmol) in $1 \mathrm{~mL}$ of dichloromethane in the dark. The solution was warmed to $\mathrm{rt}$ and stirred for $1.5 \mathrm{~h}$. Without workup, this solution was purified in the dark by flash chromatography eluting with 5\%-50\% diethyl ether in petroleum ether to afford hydroxyester 29 (48 $\mathrm{mg}, 0.17 \mathrm{mmol}, 81 \%$ ) as a yellow oily solid.

IR (neat): 3071w, 2928s, 2864m, 1810m, 1710m, 1643w, 1607w, 1576w, 1527m, 1448s, $1415 \mathrm{~m}, 1365 \mathrm{w}, 1280 \mathrm{w}, 1222 \mathrm{~m}, 1135 \mathrm{~s}, 1078 \mathrm{~m}, 1060 \mathrm{~m}, 893 \mathrm{w}, 755 \mathrm{w} \mathrm{cm}^{-1}$; ${ }^{1} \mathrm{H}-\mathrm{NMR}(500$ $\mathrm{MHz}, \mathrm{CDCl}_{3}$ ): $\delta 7.69$ (dd, $\left.J=2.0,9.0 \mathrm{~Hz}, 1 \mathrm{H}\right), 7.49$ (dd, $\left.J=1.5,7.0 \mathrm{~Hz}, 1 \mathrm{H}\right), 7.21$ (ddd, $J=1.5,7.0,8.5 \mathrm{~Hz}, 1 \mathrm{H}), 6.62$ (td, $J=2.0,7.0 \mathrm{~Hz}, 1 \mathrm{H}), 4.90$ (s, 1H), 4.74 (s, 1H), 2.97 (dd, $J=4.5,15.5 \mathrm{~Hz}, 1 \mathrm{H}), 2.85$ (dd, $J=11.0,15.5 \mathrm{~Hz}, 1 \mathrm{H}), 2.29$ (m, 1H), 2.12 (m, 1H), 1.59 (m, $1 \mathrm{H}), 1.55(\mathrm{~m}, 1 \mathrm{H}), 1.43(\mathrm{~m}, 1 \mathrm{H}), 1.27(\mathrm{~s}, 3 \mathrm{H}), 0.88(\mathrm{~s}, 3 \mathrm{H}) ;{ }^{13} \mathrm{C}-\mathrm{NMR}(125 \mathrm{MHz}$, $\left.\mathrm{CDCl}_{3}\right): \delta 178.3,168.7,148.5,137.7,137.3,133.5,112.5,109.2,49.7,37.6,35.1,33.6$, 30.7, 28.5, 24.0, 23.4. Anal. Calc'd for $\mathrm{C}_{16} \mathrm{H}_{21} \mathrm{NO}_{2} \mathrm{~S}$ : C, 65.95; H, 7.26; N, 4.81. Found: C, 66.20; H, 7.01; N, 4.62 .

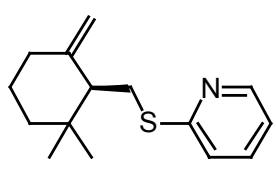

30

2-(2,2-Dimethyl-6-methylene-cyclohexylmethylsulfanyl)-pyridine $\quad$ (30): Under nitrogen to a solution of ester 29 ( $48 \mathrm{mg}, 0.165 \mathrm{mmol}$ ) in $3 \mathrm{~mL}$ of toluene was added 2 $\mathrm{mg}$ of AIBN. This mixture was heated at $93^{\circ} \mathrm{C}$ for $6 \mathrm{~h}$. The color of the mixture turned from yellow to pale yellow. The mixture was directly columned with $5 \%$ to $10 \%$ diethyl ether in petroleum ether to afford sulfide 30 (38 $\mathrm{mg}, 0.154 \mathrm{mmol}, 93 \%$ ) as a colorless oil. IR (neat): 3070w, 2928s, 2855m, 1646w, 1579s, 1556m, 1453s, 1414s, 1386w, 1366w, 1281w, 1124s, 892w, 756m, 724w cm ${ }^{-1} ;{ }^{1} \mathrm{H}-\mathrm{NMR}\left(300 \mathrm{MHz}, \mathrm{CDCl}_{3}\right): \delta 8.41(\mathrm{~m}, 1 \mathrm{H})$, $7.43(\mathrm{~m}, 1 \mathrm{H}), 7.16(\mathrm{~m}, 1 \mathrm{H}), 6.94(\mathrm{~m}, 1 \mathrm{H}), 4.87$ (s, 1H), 4.44 (s, 1H), 3.70 (dd, $J=3.6,12.6$

\footnotetext{
${ }^{3}$ Barton, D. H. R.; Fontana, G. Tetrahedron 1996, 52, 11163.
} 
$\mathrm{Hz}, 1 \mathrm{H}), 3.09$ (t, $J=12.6 \mathrm{~Hz}, 1 \mathrm{H}), 2.16(\mathrm{~m}, 3 \mathrm{H}), 1.56(\mathrm{~m}, 3 \mathrm{H}), 1.22(\mathrm{~m}, 1 \mathrm{H}), 1.04(\mathrm{~s}, 3 \mathrm{H})$, 0.96 (s, 3H); ${ }^{13} \mathrm{C}-\mathrm{NMR}\left(75 \mathrm{MHz}, \mathrm{CDCl}_{3}\right): \delta 149.3,148.0,135.6,128.4,122.2,119.0$, 109.8, 53.4, 37.4, 33.2, 29.7, 28.6, 28.1, 24.9, 23.6. HRMS: Calc'd for $\mathrm{C}_{15} \mathrm{H}_{21} \mathrm{NS}$ : 247.1395. Found: 247.1405.

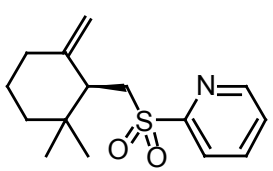

31

2-(2,2-Dimethyl-6-methylene-cyclohexylmethylsulfonyl)-pyridine (31): To a solution of 30 (25 mg, $0.10 \mathrm{mmol})$ in $0.5 \mathrm{~mL}$ of methanol at $0^{\circ} \mathrm{C}$ was added hydrogen peroxide $(0.1 \mathrm{~mL}, 30 \%$ in water) followed by sodium tungstate dehydrate $(17 \mathrm{mg}, 0.05 \mathrm{mmol})$. This mixture was warmed to rt and stirred for $6 \mathrm{~h}$. Without workup, the mixture was purified by flash chromatography eluting with $5 \%$ to $50 \%$ ethyl acetate in petroleum ether to afford sulfone 31 (21 $\mathrm{mg}, 0.075 \mathrm{mmol}, 75 \%$ ) as a sticky colorless oil, accompanied with sulfoxide ( $5 \mathrm{mg}, 0.019 \mathrm{mmol}, 19 \%)$ as a colorless oil.

IR (neat): 3067w, 2932s, 2859m, 1649w, 1579w, 1452m, 1428m, 1311s, 1167s, 1113s, 1080w, 1044w, 992w, 887m, 771 m cm ${ }^{-1}$; ${ }^{1} \mathrm{H}-\mathrm{NMR}\left(500 \mathrm{MHz}, \mathrm{CDCl}_{3}\right): \delta 8.70$ (dd, $J=2.0$, $5.0 \mathrm{~Hz}, 1 \mathrm{H}), 8.01$ (d, $J=8.0 \mathrm{~Hz}, 1 \mathrm{H}), 7.90$ (td, $J=1.5,8.0 \mathrm{~Hz}, 1 \mathrm{H}), 7.51$ (dd, $J=4.5,7.5 \mathrm{~Hz}$, $1 \mathrm{H}), 4.56(\mathrm{~s}, 1 \mathrm{H}), 4.52(\mathrm{~s}, 1 \mathrm{H}), 3.81$ (dd, $J=10.5,15.0 \mathrm{~Hz}, 1 \mathrm{H}), 3.44$ (dd, $J=2.5,15.0 \mathrm{~Hz}$, 1H), $2.48(\mathrm{~d}, J=11.0 \mathrm{~Hz}, 1 \mathrm{H}), 1.99(\mathrm{~m}, 1 \mathrm{H}), 1.87(\mathrm{~m}, 1 \mathrm{H}), 1.48(\mathrm{~m}, 2 \mathrm{H}), 1.25(\mathrm{~m}, 2 \mathrm{H})$, $1.20(\mathrm{~s}, 3 \mathrm{H}), 0.87(\mathrm{~s}, 3 \mathrm{H}) ;{ }^{13} \mathrm{C}-\mathrm{NMR}\left(125 \mathrm{MHz}, \mathrm{CDCl}_{3}\right): \delta 157.8,149.9,145.4,137.7$, 127.1, 122.4, 111.6, 49.9, 47.8, 36.4, 35.2, 31.9, 27.5, 25.6, 23.0. HRMS: Calc'd for $\mathrm{C}_{15} \mathrm{H}_{21} \mathrm{O}_{2} \mathrm{NS}\left(\mathrm{M}+\mathrm{Na}^{+}, \mathrm{ESI}\right): 302.2$. Found: 302.2 .

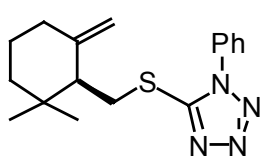

24

\section{(S)-5-(2,2-Dimethyl-6-methylene-cyclhexylmethylsulfanyl)-1-phenyl-1 $H$-tetrazole}

(24): To a solution of alcohol 21 (229 $\mathrm{mg}, 1.51 \mathrm{mmol})$ in THF $(5 \mathrm{~mL})$ were added triphenylphosphine $(475 \mathrm{mg}, 1.81 \mathrm{mmol})$ and 1-phenyl- $1 H$-tetrazole 5 -thiol $(229 \mathrm{mg}$, $1.81 \mathrm{mmol}$ ). To this solution at $0^{\circ} \mathrm{C}$ was added diisopropylazodicarboxylate $(366 \mathrm{mg}$, $0.36 \mathrm{~mL}, 1.81 \mathrm{mmol}$ ). The resulting yellow solution was warmed to $\mathrm{rt}$ and stirred for $3 \mathrm{~h}$. 
The solution was concentrated in vacuo and the residue was purified by flash chromatography eluting with $5 \%$ to $10 \%$ diethyl ether in petroleum ether to afford phenyltetrazole sulfide $\mathbf{2 4}$ (465 $\mathrm{mg}, 1.48 \mathrm{mmol}, 98 \%$ ) as a white solid.

m.p.:105-108 ${ }^{\circ} \mathrm{C} ;[\alpha]_{D}=-144^{\circ}$ (c 4.0, $\mathrm{CHCl}_{3}$ ); IR (neat): 3068s, 2937s, 2868s, $1645 \mathrm{~m}$, $1598 \mathrm{~m}, 1501 \mathrm{~m}, 1461 \mathrm{~m}, 1385 \mathrm{~s}, 1279 \mathrm{~m}, 1257 \mathrm{~m}, 1162 \mathrm{~m}, 1090 \mathrm{~m}, 1017 \mathrm{~m}, 890 \mathrm{w}, 882 \mathrm{w}$, 768w, 713w, 696m cm ${ }^{-1}$; ${ }^{1} \mathrm{H}$ NMR (300 MHz, $\mathrm{CDCl}_{3}$ ) $\delta: 7.42$ (m, 5H), 4.73 (s, 1H), 4.49 (s, 1H), 3.71 (dd, $J=3.9,12.3 \mathrm{~Hz}, 1 \mathrm{H}$ ), 3.29 (t, $J=12.3 \mathrm{~Hz}, 1 \mathrm{H}$ ), 2.10 (dd, $J=3.3,12.0 \mathrm{~Hz}$, $1 \mathrm{H}), 1.89(\mathrm{~m}, 2 \mathrm{H}), 1.37(\mathrm{~m}, 3 \mathrm{H}), 1.23(\mathrm{~m}, 1 \mathrm{H}), 0.91(\mathrm{~s}, 3 \mathrm{H}), 0.83(\mathrm{~s}, 3 \mathrm{H}) ;{ }^{13} \mathrm{C}$ NMR $(75$ $\left.\mathrm{MHz}, \mathrm{CDCl}_{3}\right) \delta: 154.8,146.6,133.6,129.8,129.6,123.6,110.8,52.8,36.5,35.7,32.3$, 32.1, 28.2, 25.2, 23.2. Anal. Calc'd for $\mathrm{C}_{17} \mathrm{H}_{22} \mathrm{~N}_{4} \mathrm{~S}: \mathrm{C}, 64.93 ; \mathrm{H}, 7.05 ; \mathrm{N}, 17.82$. Found: $\mathrm{C}, 64.93 ; \mathrm{H}, 7.01 ; \mathrm{N}, 17.70$. HPLC: OD column (Flow rate: $1.0 \mathrm{~mL} / \mathrm{min}, 99 / 1$ isopropanol/heptane, $S$ isomer: $12.68 \mathrm{~min}, R$ isomer: $16.80 \mathrm{~min}$ ).

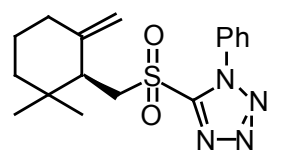

25

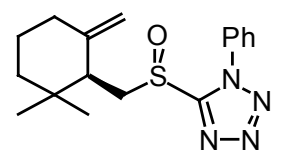

26

\section{(S)-5-(2,2-Dimethyl-6-methylene-cyclohexylmethylsulfanyl)-1-phenyl-1H-tetrazole}

(25) and (S)-5-(2,2-Dimethyl-6-methylene-cyclohexylmethylsulfinyl)-1-phenyl-1 Htetrazole (26): To a solution of phenyltetrazole sulfide $24(250 \mathrm{mg}, 0.796 \mathrm{mmol})$ in ethanol $(4 \mathrm{~mL})$ at $0^{\circ} \mathrm{C}$ were added hydrogen peroxide $(0.89 \mathrm{~mL}, 30 \%$ in water) and ammonium heptamolybdate tetrahydrate $(197 \mathrm{mg}, 0.159 \mathrm{mmol})$. The mixture was warmed to $\mathrm{rt}$ and stirred overnight. Without workup, the reaction mixture was directly purified by silica gel chromatography eluting with $5 \%$ to $15 \%$ diethyl ether in petroleum ether to afford sulfone $25(185 \mathrm{mg}, 0.53 \mathrm{mmol}, 67 \%)$ and sulfoxide $26(85 \mathrm{mg}, 0.26$ mmol, 32\%). The sulfoxide was resubmitted to the reaction and stirred overnight. To a solution of sulfoxide $26(85 \mathrm{mg}, 0.26 \mathrm{mmol})$ in ethanol $(3 \mathrm{~mL})$ at $0^{\circ} \mathrm{C}$ were added hydrogen peroxide $(0.40 \mathrm{~mL}, 30 \%$ in water) and ammonium heptamolybdate tetrahydrate (90 $\mathrm{mg}, 0.0 .073 \mathrm{mmol}$ ). The resulting lemonade yellow slurry was stirred at $\mathrm{rt}$ overnight. The mixture was purified by column chromatography eluting with $5 \%$ to $10 \%$ diethyl ether in petroleum ether to afford sulfone $\mathbf{2 5}(88 \mathrm{mg}, 0.25 \mathrm{mmol}, 98 \%)$ as a colorless oil. 
25: $[\alpha]_{D}=-45.0^{\circ}$ (c 4.2, $\mathrm{CHCl}_{3}$ ); IR (neat): $3075 \mathrm{~m}, 2935 \mathrm{~s}, 2870 \mathrm{~s}, 1649 \mathrm{~m}, 1596 \mathrm{~m}, 1498 \mathrm{~s}$, $1461 \mathrm{~m}, 1391 \mathrm{w}, 1353 \mathrm{~s}, 1264 \mathrm{w}, 1229 \mathrm{w}, 1149 \mathrm{~s}, 1104 \mathrm{~m}, 1076 \mathrm{~m}, 1046 \mathrm{~m}, 1016 \mathrm{w}, 971 \mathrm{w}$, 902m, 763s, 690s, 618 $\mathrm{sm}^{-1}$; ${ }^{1} \mathrm{H}$ NMR (300 MHz, $\left.\mathrm{CDCl}_{3}\right) \delta: 7.58(\mathrm{~m}, 5 \mathrm{H}), 4.74(\mathrm{~s}, 1 \mathrm{H})$, 4.60 (s, 1H), 4.04 (dd, $J=11.1,14.7 \mathrm{~Hz}, 1 \mathrm{H}), 3.86$ (dd, $J=2.7,14.7 \mathrm{~Hz}, 1 \mathrm{H}), 2.60$ (dd, $J=2.1,10.8 \mathrm{~Hz}, 1 \mathrm{H}), 2.17(\mathrm{~m}, 1 \mathrm{H}), 2.00(\mathrm{~m}, 1 \mathrm{H}), 1.59$ (m, 2H), 1.35 (m, 2H), 0.97 (s,

$3 \mathrm{H}), 0.91(\mathrm{~s}, 3 \mathrm{H}) ;{ }^{13} \mathrm{C}$ NMR $\left(75 \mathrm{MHz}, \mathrm{CDCl}_{3}\right) \delta: 153.8,145.4,133.0,131.3,129.5$, 125.2, 111.9, 54.6, 47.2, 36.1, 35.2, 31.7, 27.3, 26.1, 22.8. HRMS: Calc'd for $\mathrm{C}_{17} \mathrm{H}_{22} \mathrm{O}_{2} \mathrm{~N}_{4} \mathrm{~S}: 346.1469$. Found: 346.1463 .

26: $[\alpha]_{D}=+15.7^{\circ}$ ( c 2.0, $\mathrm{CHCl}_{3}$ ); IR (neat): 3360b, 3073w, 2934s, 2869s, 1731w, 1646w, $1596 \mathrm{w}, 1498 \mathrm{~s}, 1463 \mathrm{~m}, 1386 \mathrm{~m}, 1368 \mathrm{~m}, 1228 \mathrm{w}, 1148 \mathrm{w}, 1068 \mathrm{~s}, 1015 \mathrm{~m}, 903 \mathrm{w}, 763 \mathrm{~s}, 690 \mathrm{~s}$ $\mathrm{cm}^{-1}$; ${ }^{1} \mathrm{H}$ NMR $\left(300 \mathrm{MHz}, \mathrm{CDCl}_{3}\right)$ ): $7.65(\mathrm{~m}, 5 \mathrm{H}), 4.92(\mathrm{~s}, 1 \mathrm{H}), 4.70(\mathrm{~s}, 1 \mathrm{H}), 3.90$ (dd, $J=3.3,13.2 \mathrm{~Hz}, 1 \mathrm{H}), 3.47$ (t, $J=13.2 \mathrm{~Hz}, 1 \mathrm{H}), 2.54$ (dd, $J=3.0,12.9 \mathrm{~Hz}, 1 \mathrm{H}), 2.05$ (m, $2 \mathrm{H}), 1.54(\mathrm{~m}, 2 \mathrm{H}), 1.26(\mathrm{~m}, 2 \mathrm{H}), 1.24(\mathrm{~s}, 3 \mathrm{H}), 1.01(\mathrm{~s}, 3 \mathrm{H}) ;{ }^{13} \mathrm{C} \mathrm{NMR}\left(75 \mathrm{MHz}, \mathrm{CDCl}_{3}\right)$ $\delta: 156.7,145.1,133.0,131.1,129.9,125.0,112.4,52.2,46.8,36.4,35.2,32.1,27.8,25.7$, 23.1.

\section{(R)-Trifluoromethanesulfonic acid 5-methoxy-2,7-dimethyl-chroman-2-ylmethyl} ester (32): To a solution of crude aldehyde $\mathbf{1 6 b}$ (320 mg, $1.45 \mathrm{mmol})$ in diethyl ether (10 $\mathrm{mL})$ at $\mathrm{rt}$ was added solid $\mathrm{LiAlH}_{4}(0.095 \mathrm{mmol}, 2.5 \mathrm{mmol})$. The mixture was stirred at $\mathrm{rt}$ for $15 \mathrm{~min}$, and to this slurry was added a saturated solution of $\mathrm{Na}_{2} \mathrm{SO}_{4}$ (10 drops). The resulting solution was stirred for $15 \mathrm{~min}$, and filtered through a pad of silica gel washing with diethyl ether. After evaporation, the residue was chromatographed using diethyl ether/petroleum ether $(\mathrm{v} / \mathrm{v}=1 / 5)$ to afford alcohol $(0.300 \mathrm{~g}, 1.35 \mathrm{mmol})$ as a colorless oil. IR (neat): 3417, 2936, 1618, 1586, 1463, $1109 \mathrm{~cm}^{-1} ;{ }^{1} \mathrm{H}$ NMR (500 MHz, $\left.\mathrm{CDCl}_{3}\right) \delta: 6.34$ (s, 1H), 6.29 (s, 1H), 3.84 (s, 3H), 3.66 (dd, $J=6.4,11.4 \mathrm{~Hz}, 1 \mathrm{H}), 3.62$ (dd, $J=6.8,11.4$ Hz, 1H), 2.76 (ddd, $J=4.9,6.1,17.3 \mathrm{~Hz}, 1 \mathrm{H}), 2.60$ (ddd, $J=6.3,10.2,16.8 \mathrm{~Hz}, 1 \mathrm{H}), 2.40$ (t, $J=6.6 \mathrm{~Hz}, 1 \mathrm{H}), 2.31$ (s, 3H), 1.98 (ddd, $J=6.1,10.2,13.4 \mathrm{~Hz}, 1 \mathrm{H}$ ), 1.73 (ddd, $J=4.6$, 6.6, $13.6 \mathrm{~Hz}, 1 \mathrm{H}), 1.28(\mathrm{~s}, 3 \mathrm{H}) ;{ }^{13} \mathrm{C} \mathrm{NMR}\left(125 \mathrm{MHz}, \mathrm{CDCl}_{3}\right) \delta$ : 157.5, 153.6, 137.0, 110.1, 106.9, 102.7, 76.0, 68.9, 55.2, 27.0, 21.5, 20.2, 15.9. Anal. Calc'd for $\mathrm{C}_{13} \mathrm{H}_{18} \mathrm{O}_{3}: \mathrm{C}$, 
70.24; H, 8.16. Found: C, 70.36; H, 7.96. HRMS: Calc'd for $\mathrm{C}_{13} \mathrm{H}_{18} \mathrm{O}_{3}\left[\mathrm{M}^{+}\right]: 222.1256$. Found: 222.1249 .

To an ice-cooled solution of alcohol $(0.300 \mathrm{~g}, 1.35 \mathrm{mmol})$ in dichloromethane $(14 \mathrm{~mL})$ was added 2,6-di-t-butyl-4-methylpyridine $(0.562 \mathrm{~g}, 2.73 \mathrm{mmol})$ and dropwise triflic anhydride $(0.44 \mathrm{~mL}, 2.61 \mathrm{mmol})$. The solution was stirred at $0^{\circ} \mathrm{C}$ for $20 \mathrm{~min}$. To this solution was then added water $(10 \mathrm{~mL})$. The aqueous layer was extracted with dichloromethane $(2 \times 15 \mathrm{~mL})$. The combined organic extracts were washed with brine (15 $\mathrm{mL}$ ) and dried over magnesium sulfate. After evaporation, the residue was resuspended in a mixture petroleum ether/diethyl ether $(3 / 1 \mathrm{v} / \mathrm{v}$ and quickly filtered through a pad of silica gel (petroleum ether/diethyl ether $=3 / 1 \mathrm{v} / \mathrm{v}$ ). The filtrate was concentrated in vacuo, and the residue was purified on silica gel (petroleum ether/diethyl ether $=9 / 1 \mathrm{v} / \mathrm{v}$ ) to afford triflate $32(0.420 \mathrm{~g}, 1.18 \mathrm{mmol})$ as a greenish oil.

IR (neat): 2942, 1620, 1588, 1245, 1209, $1109 \mathrm{~cm}^{-1} ;{ }^{1} \mathrm{H}$ NMR $\left(500 \mathrm{MHz}, \mathrm{CDCl}_{3}\right) \delta: 6.35$ (s, 1H), $6.31(\mathrm{~s}, 1 \mathrm{H}), 4.50(\mathrm{~d}, J=10.0 \mathrm{~Hz}, 1 \mathrm{H}), 4.45(\mathrm{~d}, J=10.0 \mathrm{~Hz}, 1 \mathrm{H}), 3.84(\mathrm{~s}, 3 \mathrm{H})$, $2.72(\mathrm{td}, J=6.1,17.3 \mathrm{~Hz}, 1 \mathrm{H}), 2.65(\mathrm{ddd}, J=6.8,9.0,17.3 \mathrm{~Hz}, 1 \mathrm{H}), 2.32(\mathrm{~s}, 3 \mathrm{H}), 1.98$ (ddd, $J=6.3,8.5,13.6 \mathrm{~Hz}, 1 \mathrm{H}), 1.84(\mathrm{td}, J=6.3,13.4 \mathrm{~Hz}, 1 \mathrm{H}), 1.40(\mathrm{~s}, 3 \mathrm{H}) ;{ }^{13} \mathrm{C} \mathrm{NMR}$ $\left(125 \mathrm{MHz}, \mathrm{CDCl}_{3}\right) \delta: 157.4,152.8,137.6,118.5\left(\mathrm{~J}_{\mathrm{CF}}=319 \mathrm{~Hz}\right), 110.22,106.1,103.3,79.3$,

73.4, 55.3, 27.2, 21.5, 20.9, 15.6. HRMS: Calc'd for $\mathrm{C}_{14} \mathrm{H}_{17} \mathrm{~F}_{3} \mathrm{O}_{5} \mathrm{~S}\left[\mathrm{M}^{+}\right]$: 354.0749. Found: 354.0747.

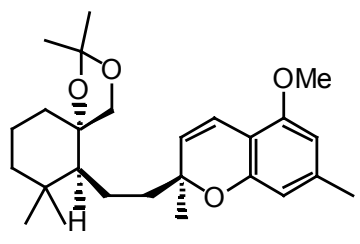

39

(2S)-5-Methoxy-2,7-dimethyl-2-[(5S,6S)-2-(2,2,7,7-tetramethyl-1,3-dioxa-spiro[4.5]dec-6-yl)-ethyl]-2H-chromene (39): To a solution of diol 38 (0.22 g, $0.59 \mathrm{mmol})$ and CSA (4 mg) in dichloromethane $(5 \mathrm{~mL})$ was added dropwise 2-methoxypropene $(0.2 \mathrm{~mL})$ (CAUTION : the end of the needle has to be immersed in the solution during the addition). The solution was stirred for $5 \mathrm{~min}$ and a saturated solution of sodium bicarbonate $(5 \mathrm{~mL})$ was added. After separation, the aqueous phase was extracted with dichloromethane $(3 \times 20 \mathrm{~mL})$. The combined organic extracts were dried over 
magnesium sulfate and then concentrated in vacuo. The residue was purified over silica gel eluting with 1/10 diethyl ether in petroleum ether to afford acetonide 39 ( $0.24 \mathrm{~g}, 0.58$ mmol, 98\%) as a colorless oil.

$[\alpha]_{\mathrm{D}}=+55.0^{\circ}\left(c 0.90, \mathrm{CHCl}_{3}\right)$; IR (neat): 2935s, 2867s, 1635m, 1614s, $1574 \mathrm{~s}, 1463 \mathrm{~s}$, $1388 \mathrm{~m}, 1378 \mathrm{~m}, 1367 \mathrm{~s}, 1332 \mathrm{w}, 1249 \mathrm{~m}, 1232 \mathrm{~s}, 1215 \mathrm{~s}, 1142 \mathrm{~s}, 1215 \mathrm{~s}, 1054 \mathrm{~s}, 1024 \mathrm{~m}, 924 \mathrm{w}$, 891w, 876w, 814w, 776w, 708w cm ${ }^{-1}$; ${ }^{1} \mathrm{H}$ NMR (500 MHz, $\left.\mathrm{CDCl}_{3}\right) \delta: 6.66$ (d, J=10.0 Hz, $1 \mathrm{H}), 6.28(\mathrm{~s}, 1 \mathrm{H}), 6.22(\mathrm{~s}, 1 \mathrm{H}), 5.46(\mathrm{~d}, J=10.0 \mathrm{~Hz}, 1 \mathrm{H}), 3.81$ (s, 3H), 3.80 (d, J=8.3 Hz, 1H), $2.28(\mathrm{~s}, 3 \mathrm{H}), 1.99(\mathrm{~m}, 1 \mathrm{H}), 1.87$ (td, $J=5.6,13.6 \mathrm{~Hz}, 1 \mathrm{H}), 1.78(\mathrm{td}, J=4.4,13.6 \mathrm{~Hz}$, 1H), 1.68-1.50 (m, 3H), 1.50-1.20 (m, 2H), 1.44 (s, 3H), 1.35 (s, 6H), $0.98(\mathrm{~s}, 3 \mathrm{H}), 0.68$ $(\mathrm{s}, 3 \mathrm{H}) ;{ }^{13} \mathrm{C} \mathrm{NMR}\left(125 \mathrm{MHz}, \mathrm{CDCl}_{3}\right) \delta: 155.0,153.9,139.2,126.6,117.2,109.8,107.6$, $106.9,103.6,85.5,68.3,55.5,53.2,44.8,40.6,38.0,35.9,31.4,28.75,28.73,26.9,26.5$, 21.9, 20.5, 20.4. HMRS: Calc'd for $\mathrm{C}_{26} \mathrm{H}_{38} \mathrm{O}_{4}\left[\mathrm{M}^{+}\right]$: 414.2770. Found: 414.2772.

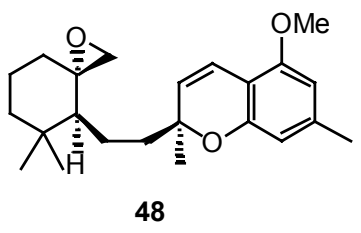

(3S,4S)-2-[2-(5,5-Dimethyl-1-oxa-spiro[2.5]oct-4-yl)-ethyl]-(2S)-5-methoxy-2,7-

dimeth-yl-2H-chroman (48): To a solution of diol $38(0.220 \mathrm{~g}, 0.588 \mathrm{mmol})$ in $5 \mathrm{~mL}$ of dichloromethane was added successively at $\mathrm{rt}$ triethyl amine $(0.170 \mathrm{~mL}, 1.21 \mathrm{mmol})$, DMAP $(0.15 \mathrm{~g}, 1.22 \mathrm{mmol})$ and $p$ - $\operatorname{TsCl}(0.168 \mathrm{~g}, 0.88 \mathrm{mmol})$. The reaction was stirred at $\mathrm{rt}$ for $3 \mathrm{~h}$. Without workup, the mixture was directly purified by preparative TLC (first eluting with diethyl ether in petroleum ether (1:1), second elution if necessary using diethyl ether in petroleum ether $(2: 1)$ to afford pure tosylate $(0.277 \mathrm{~g}, 0.524 \mathrm{mmol})$. The material was dissolved in THF (10 mL) and DMSO (15 drops). Sodium hydride (excess) was added, and the reaction mixture was stirred at $\mathrm{rt}$ for $10 \mathrm{~min}$. The mixture was directly filtered through a pad of silica gel washing with diethyl ether. The filtrate was concentracted in vacuo, and the oil material was purified on silica gel eluting with diethyl ether in petroleum ether (1/9 to $2 / 3)$ to afford epoxide $48(0.195 \mathrm{~g}, 0.546 \mathrm{mmol}, 93 \%)$ as a colorless oil.

$[\alpha]_{\mathrm{D}}=+50.2^{\circ}\left(c 1.19, \mathrm{CHCl}_{3}\right)$; IR (neat): 2937, 1614, 1573, 1463, $1119 \mathrm{~cm}^{-1} ;{ }^{1} \mathrm{H}-\mathrm{NMR}$ $\left(500 \mathrm{MHz}, \mathrm{CDCl}_{3}\right): \delta 6.67(\mathrm{~d}, J=10.0 \mathrm{~Hz}, 1 \mathrm{H}), 6.29(\mathrm{~s}, 1 \mathrm{H}), 6.24(\mathrm{~s}, 1 \mathrm{H}), 5.45(\mathrm{~d}, J=10.0$ 
$\mathrm{Hz}, 1 \mathrm{H}), 3.82(\mathrm{~s}, 3 \mathrm{H}), 2.63$ (d, J=4.7 Hz, 1H), 2.49 (dd, $J=1.2,4.6 \mathrm{~Hz}, 1 \mathrm{H}), 2.29(\mathrm{~s}, 3 \mathrm{H})$, 1.78-1.56 (m, 6H), $1.46(\mathrm{~m}, 1 \mathrm{H}), 1.34(\mathrm{~s}, 3 \mathrm{H}), 1.34(\mathrm{~m}$, overlapped, $1 \mathrm{H}), 1.24(\mathrm{~m}, 2 \mathrm{H})$, $1.09(\mathrm{~m}, 1 \mathrm{H}), 1.01(\mathrm{~s}, 3 \mathrm{H}), 0.84(\mathrm{~s}, 3 \mathrm{H}) ;{ }^{13} \mathrm{C}-\mathrm{NMR}\left(125 \mathrm{MHz}, \mathrm{CDCl}_{3}\right): \delta$ 155.7, 154.4, $140.1,127.3,118.1,110.6,108.5,104.5,79.0,60.0,55.5,51.3,50.2,41.4,36.9$, 35.7, 31.9, 30.3, 28.8, 26.3, 22.0, 21.0, 20.6. HRMS: Calc'd for $\mathrm{C}_{23} \mathrm{H}_{32} \mathrm{O}_{3}$ : 356.2351. Found: 356.2360 .

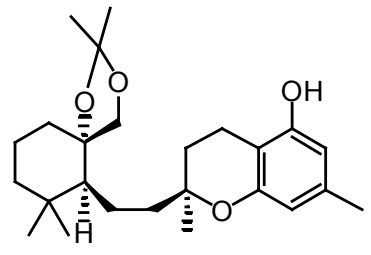

69

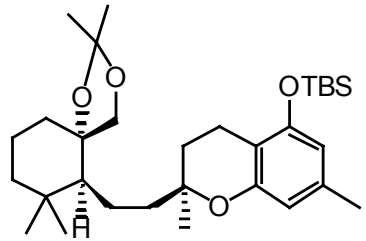

70

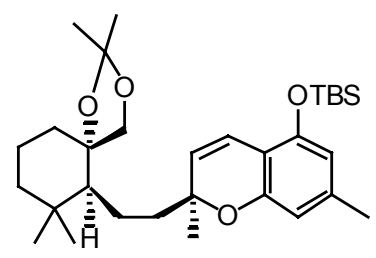

71

(-)-Siccanochromene $\mathbf{E}$ diacetate (69), 12-acetoxy-12b-acetoxymethyl-4,4,6a,9tetramethyl-1,3,4,4a,5,6,6a,12,12a,12b-deca-hydro- $2 H$-benzo[a]xanthen-11-yl acetate (70) and 11,12-Dihydroxy-4,4,6a,9-tetramethyl-1,3,4,4a,5,6,6a,12,12adecahydrobenzo[a]-xanthen-12b-ylmethyl acetate (71): To a solution of (-)-siccanin (90 mg, $0.26 \mathrm{mmol})$ in $1.2 \mathrm{~mL}$ of dichloromethane was added acetic anhydride $(1.2 \mathrm{~mL})$. To this solution at $0^{\circ} \mathrm{C}$ was added borontrifluoride etherate $(0.025 \mathrm{~mL})$. The resulting blue solution was stirred at $0^{\circ} \mathrm{C}$ for $3 \mathrm{~min}$ and the color remained. This mixture was then quenched a piece of ice to afford a colorless solution. The mixture was extracted with ethyl acetate $(3 \times 10 \mathrm{~mL})$. The combined organic extracts were dried with magnesium sulfate and concentrated in vacuo. The residue was purified by flash chromatography eluting with $5 \%$ to $70 \%$ of diethyl ether in petroleum ether to separate three products: 69 (18 mg, $0.042 \mathrm{mmol}, 16 \%$ ) as a colorless oil, 70 (64 mg, $0.13 \mathrm{mmol}, 50 \%$ ) as a white oily solid, and 71 (26 mg, $0.066 \mathrm{mmol}, 25 \%$ ) as a brown oil.

69: $[\alpha]_{\mathrm{D}}=-62.3^{\circ}\left(c 0.69, \mathrm{CDCl}_{3}\right)$; IR (neat): 2929s, $1770 \mathrm{~s}, 1740 \mathrm{~s}, 1625 \mathrm{~m}, 1568 \mathrm{w}$, $1455 \mathrm{~m}, 1315 \mathrm{w}, 1233 \mathrm{~s}, 1197 \mathrm{~s}, 1128 \mathrm{w}, 1083 \mathrm{w}, 1056 \mathrm{~m}, 859 \mathrm{w} \mathrm{cm}{ }^{-1}$; ${ }^{1} \mathrm{H}-\mathrm{NMR}(500 \mathrm{MHz}$, $\left.\mathrm{CDCl}_{3}\right): \delta 6.58(\mathrm{~s}, 1 \mathrm{H}), 6.48(\mathrm{~d}, J=0.5 \mathrm{~Hz}, 1 \mathrm{H}), 6.30(\mathrm{~s}, 1 \mathrm{H}), 4.10(\mathrm{~d}, J=11.0 \mathrm{~Hz}, 1 \mathrm{H})$, $3.91(\mathrm{~d}, J=11.0 \mathrm{~Hz}, 1 \mathrm{H}), 2.37(\mathrm{~m}, 1 \mathrm{H}), 2.35$ (s, 3H), 2.30 (s, 3H), 2.05 (s, 3H), 1.95 (m, 2H), $1.87(\mathrm{~m}, 1 \mathrm{H}), 1.58(\mathrm{~m}, 2 \mathrm{H}), 1.47(\mathrm{~m}, 1 \mathrm{H}), 1.41(\mathrm{~m}, 3 \mathrm{H}), 1.38(\mathrm{~s}, 3 \mathrm{H}), 1.06(\mathrm{~s}, 3 \mathrm{H})$, $1.01(\mathrm{~s}, 3 \mathrm{H}) ;{ }^{13} \mathrm{C}-\mathrm{NMR}\left(125 \mathrm{MHz}, \mathrm{CDCl}_{3}\right): \delta 171.2,169.1,152.5,145.9,139.2,138.9$, $114.9,114.7,114.1,113.8,71.3,44.5,43.8,42.4,35.8,34.3,32.49,32.46,27.5,26.4$, 
21.5, 20.8, 20.7, 19.0, 17.8. Anal. Calc'd for $\mathrm{C}_{26} \mathrm{H}_{34} \mathrm{O}_{5}: \mathrm{C}, 73.21$; H, 8.03. Found: $\mathrm{C}$, $72.98 ; \mathrm{H}, 8.29$.

70: $[\alpha]_{D}=-65.6^{\circ}$ (c 2.9, $\mathrm{Et}_{2} \mathrm{O}$ ); IR (neat): 2928m, 1764m, 1737s, $1630 \mathrm{~m}, 1582 \mathrm{~m}, 1498 \mathrm{w}$, 1456m, 1370m, 1324w, 1246s, 1202s, 1169m, 1140w, 1041m, 959w, 910m, 861w, 733m $\mathrm{cm}^{-1} ;{ }^{1} \mathrm{H}-\mathrm{NMR}\left(500 \mathrm{MHz}, \mathrm{CDCl}_{3}\right): \delta 6.59(\mathrm{~s}, 1 \mathrm{H}), 6.49(\mathrm{~s}, 1 \mathrm{H}), 5.96(\mathrm{~s}, 1 \mathrm{H}), 4.58(\mathrm{~d}$, $J=12.5 \mathrm{~Hz}, 1 \mathrm{H}), 4.49$ (d, $J=12.5 \mathrm{~Hz}, 1 \mathrm{H}), 2.31$ (s, 3H), 2.23 (s, 3H), 2.10 (s, 3H), 2.06 (m, 1H), $2.02(\mathrm{~s}, 3 \mathrm{H}), 1.75(\mathrm{~m}, 2 \mathrm{H}), 1.20-1.60(\mathrm{~m}, 7 \mathrm{H}), 1.05(\mathrm{~s}, 3 \mathrm{H}), 0.93$ (s, 3H), 0.89 (m, $1 \mathrm{H}), 0.78(\mathrm{~d}, J=12.0 \mathrm{~Hz}, 1 \mathrm{H}) ;{ }^{13} \mathrm{C}-\mathrm{NMR}\left(125 \mathrm{MHz}, \mathrm{CDCl}_{3}\right): \delta 170.9,169.6,169.3,155.1$, 149.7, 140.5, 115.7, 115.1, 110.8, 74.9, 66.9, 63.0, 47.4, 43.9, 41.0, 39.1, 34.2, 33.8, 33.6, 29.7, 27.6, 24.1, 21.4, 21.0, 20.9, 20.8, 19.6, 18.5. Anal. Calc'd for $\mathrm{C}_{28} \mathrm{H}_{38} \mathrm{O}_{7}$ : C, 69.11; H, 7.87. Found: C, 68.89; H, 8.01.

71: $[\alpha]_{\mathrm{D}}=-46.5^{\circ}$ (c 2.9, Et $2 \mathrm{O}$ ); IR (neat): 3358b, 2926s, 1711s, $1627 \mathrm{~m}, 1592 \mathrm{~m}, 1492 \mathrm{w}$, 1457 w, 1438w, 1393w, 1361w, 1264s, 1202w, 1170w, 1141w, 1121w, 1078m, 1038s, 1012w, 909m, 825w, 734s cm ${ }^{-1}$; ${ }^{1} \mathrm{H}-\mathrm{NMR}\left(500 \mathrm{MHz}, \mathrm{CDCl}_{3}\right.$ ): $\delta 6.32(\mathrm{~s}, 1 \mathrm{H}), 6.28$ (s, 1H), 4.91 (s, 1H), 4.66 (d, $J=12.5 \mathrm{~Hz}, 1 \mathrm{H}), 4.34$ (d, $J=12.5 \mathrm{~Hz}, 1 \mathrm{H}), 2.24$ (s, 3H), 2.21 (s, $3 \mathrm{H}), 2.07(\mathrm{~m}, 2 \mathrm{H}), 1.75(\mathrm{~m}, 1 \mathrm{H}), 1.58(\mathrm{~m}, 1 \mathrm{H}), 1.50(\mathrm{~m}, 3 \mathrm{H}), 1.10-1.25(\mathrm{~m}, 3 \mathrm{H}), 1.27(\mathrm{~s}$, $3 \mathrm{H}), 1.13$ (s, 3H), $0.92(\mathrm{~s}, 3 \mathrm{H}), 0.88(\mathrm{~m}, 1 \mathrm{H}), 0.63(\mathrm{~d}, J=14.0 \mathrm{~Hz}, 1 \mathrm{H}) ;{ }^{13} \mathrm{C}-\mathrm{NMR}(125$ $\mathrm{MHz}, \mathrm{CDCl}_{3}$ ): $\delta 173.3,155.9,153.7,139.8,109.6,108.6,108.2,74.4,66.6,62.3,50.2$, 44.1, 41.2, 38.8, 34.1, 33.74, 33.68, 29.7, 26.7, 23.5, 21.4, 21.4, 19.4, 18.0. Anal. Calc'd for $\mathrm{C}_{24} \mathrm{H}_{34} \mathrm{O}_{5}$ : C, 71.61; H, 8.51. Found: C, 71.97; H, 8.10.

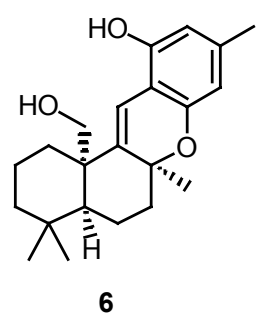

(-)-Siccanochromene E (6): Method A: To a solution of diacetate 69 (15 mg, 0.035 mmol) in $1 \mathrm{~mL}$ of dichloromethane at $-78^{\circ} \mathrm{C}$ was added DIBAL-H $(0.16 \mathrm{~mL}, 0.16 \mathrm{mmol}$, $1 \mathrm{M}$ in heptane). This mixture was slowly warmed to $\mathrm{rt}$ over $10 \mathrm{~min}$. The mixture was quenched with a few drops of water, and without further workup, directly purified by flash chromatography eluting with $10 \%$ to $60 \%$ diethyl ether in petroleum ether to afford 6 (11.5 mg, $0.033 \mathrm{mmol}, 95 \%)$ as a white solid. 
Method B: To a solution of triacetate $70(15 \mathrm{mg}, 0.031 \mathrm{mmol})$ in $1 \mathrm{~mL}$ of dichloromethane at $-78^{\circ} \mathrm{C}$ was added borontrifluoride etherate $(0.05 \mathrm{~mL})$. After $20 \mathrm{~min}$, this solution was warmed to $\mathrm{rt}$ and filtered through a pad of silica gel. To the filtrate at $0^{\circ} \mathrm{C}$ was added DIBAL-H $(0.12 \mathrm{~mL}, 0.12 \mathrm{mmol}, 1 \mathrm{M}$ in hexane $)$, the resulting mixture was stirred for $30 \mathrm{~min}$. Without workup, the mixture was directly purified by flash chromatography eluting with $20 \%$ diethyl ether in petroleum ether to afford $6(8.7 \mathrm{mg}$, $0.025 \mathrm{mmol}, 82 \%$ ) as a white solid.

$[\alpha]_{D}=-80^{\circ} \quad(c \quad 0.8$, EtOH $) ;[\alpha]^{\text {lit }}=-86^{\circ}$ (c unspecified, EtOH $) ;^{2 b}$ m.p. $=178-180^{\circ} \mathrm{C}$, m.p. $=188^{\circ} \mathrm{C}^{\text {lit }}{ }^{2 \mathrm{~b}}$ IR (neat): $3336 \mathrm{~b}, 2932 \mathrm{~s}, 2868 \mathrm{~m}, 1625 \mathrm{~s}, 1580 \mathrm{w}, 1510 \mathrm{w}, 1454 \mathrm{~m}, 1388 \mathrm{w}$, $1361 \mathrm{w}, 1324 \mathrm{~m}, 1297 \mathrm{w}, 1275 \mathrm{w}, 1234 \mathrm{w}, 1198 \mathrm{~m}, 1129 \mathrm{~s}, 1098 \mathrm{~s}, 1065 \mathrm{~s}, 1062 \mathrm{~s}, 994 \mathrm{~m}$, 910w, 826w, 733m cm ${ }^{-1}$; ${ }^{1} \mathrm{H}-\mathrm{NMR}\left(500 \mathrm{MHz}, \mathrm{CDCl}_{3}\right): \delta 6.58(\mathrm{~s}, 1 \mathrm{H}), 6.27(\mathrm{~s}, 1 \mathrm{H}), 6.19$ (s, 1H), $5.83(\mathrm{~s}, 1 \mathrm{H}), 3.76(\mathrm{~d}, J=10.5 \mathrm{~Hz}, 1 \mathrm{H}), 3.28$ (t, $J=9.5 \mathrm{~Hz}, 1 \mathrm{H}), 2.42$ (d, J=14.0 Hz, 1H), $2.34(\mathrm{td}, J=7.5,13.0 \mathrm{~Hz}, 1 \mathrm{H}), 2.21(\mathrm{~s}, 3 \mathrm{H}), 1.90(\mathrm{~m}, 2 \mathrm{H}), 1.79(\mathrm{~m}, 1 \mathrm{H}), 1.68$ (d, $J=7.5 \mathrm{~Hz}, 1 \mathrm{H}), 1.51(\mathrm{~m}, 1 \mathrm{H}), 1.39$ (s, 3H), 1.40-1.21 (m, 5H), 1.03 (s, 3H), $0.96(\mathrm{~s}, 3 \mathrm{H})$; ${ }^{13} \mathrm{C}-\mathrm{NMR}\left(125 \mathrm{MHz}, \mathrm{CDCl}_{3}\right): \delta 152.8,151.0,139.4,136.8,115.0,109.7,109.0,108.8$, 76.4, 70.9, 45.7, 44.4, 43.7, 36.1, 34.4, 32.5, 32.3, 27.1, 26.4, 21.6, 19.2, 17.8. Anal. Calc'd for $\mathrm{C}_{22} \mathrm{H}_{30} \mathrm{O}_{3}$ : C, 77.16; $\mathrm{H}, 8.83$. Found: C, 77.28; $\mathrm{H}, 8.70$. 